Proceedings of the 46th International School and Conference on the Physics of Semiconductors "Jaszowiec" 2017, Szczyrk

\title{
Coupling of Quantum Dots with Quantum Wells in a System Based on (Cd,Zn,Mg)Te
}

\author{
K. Polczyńska, E. Janik, P. Kossacki and W. Pacuski* \\ Institute of Experimental Physics, Faculty of Physics, University of Warsaw, \\ L. Pasteura 5, PL-02-093 Warsaw, Poland
}

\begin{abstract}
Coupled low dimensional structures have potential applications in quantum computing and spintronics. Using molecular beam epitaxy we fabricated three kinds of systems of coupled quantum wells and quantum dots with different energy order: wells at higher energy than dots, resonant structures, and dots at higher energy than wells. By analysis of photoluminescence and reflectivity spectra, we conclude that there is a possibility of effective carrier tunneling between structures, which opens possibility of subsequent testing of spin transfer efficiency.
\end{abstract}

DOI: 10.12693/APhysPolA.132.369

PACS/topics: 78.55.Et, 78.67.Hc, 73.21.La, 78.67.--n

\section{Introduction}

Coupled quantum systems are desirable structures due to potential application in spintronics and quantum computing $[1,2]$. Particularly interesting are coupled objects with different dimensionality [3], like quantum wells (QWs) - 2D structures, and quantum dots (QDs) $0 \mathrm{D}$ structures $[4,5]$. Thanks to combining such different objects in one structure, one can think about unusual combination of physical properties. For example, spin relaxation in QWs, and in particular semimagnetic QWs [6-8], is very fast, but it is rather slow in QDs [9] or QDs containing single magnetic ions [10,11]. Therefore structure with coupled QWs and QDs could be used for efficient spin orientation of carriers in QWs and further injection of such polarized carriers to QD, where spin will be preserved long time. Polarization of carriers in QDs can be subsequently transferred to magnetic ions, and in particular to single magnetic ion in a QD [12-17].

However, there are important problems in designing coupled quantum structures with different dimensionality. For resonant tunneling QW energy should be similar to QDs energy, but due to quantum confinement, larger objects like QWs exhibit typically lower energy than smaller object like QDs, if they are made of the same semiconductor. Moreover, QWs should not be strained too much, while QDs typically require strain for the formation process. Therefore, it is difficult to find good material combination for realization of coupled QWs and QDs. In particular, in telluride systems, good optical properties can be obtained for CdTe QWs embedded in $(\mathrm{Cd}, \mathrm{Mg}) \mathrm{Te}$ barriers, and $\mathrm{CdTe}$ QDs in $\mathrm{ZnTe}$ barriers. However emission energy of such QWs is about $1.6 \mathrm{eV}$ [18], and about $2 \mathrm{eV}$ for QDs [19]. Moreover ZnTe barrier exhibit much smaller lattice parameter than $\mathrm{CdTe}$ or $(\mathrm{Cd}, \mathrm{Mg}) \mathrm{Te}$.

*corresponding author; e-mail: Wojciech.Pacuski@fuw.edu.pl
Here we propose three Te-based systems of coupled QWs and QDs. In the first, QW has slightly higher energy than QDs, in the second, both are close to resonance, and in the third system, QDs are at slightly higher energy than QWs.

\section{Results}

Samples were grown using molecular beam epitaxy (MBE) on quarters of 3 inch GaAs:Si (100)-oriented substrates. We present two samples for each system to show the difference between spectra of QWs and QDs. We measured microphotoluminescence and reflectivity spectra obtained at low temperature $(T=8 \mathrm{~K})$.

In the first system $\mathrm{QW}$ is made of $(\mathrm{Cd}, \mathrm{Mn}, \mathrm{Mg}) \mathrm{Te}$ material with low $\mathrm{Mg}$ content, barrier is made of $(\mathrm{Cd}, \mathrm{Mg}) \mathrm{Te}$ with higher Mg content, and QDs are made of CdTe:Mn (see scheme in Fig. 1a). Adding $\mathrm{Mg}$ to $\mathrm{QW}$ is not a typical procedure, but opens possibility to increase QW energy. We made two comparable samples. The first contains three QWs. One of them contains QDs layer in the middle. The second sample contains only one QW with QDs inside. The photoluminescence (PL) spectra (Fig. 1b) shows two peaks for the first sample. They are related to QWs and QDs, respectively. The second sample exhibits no peak of QW. Moreover, QDs peak has higher intensity than the same signal from the sample with more QWs. This means that all of the carriers from QW are efficiently relaxing to QDs, as it was predicted. QW peak is spectrally wide, its full width at half maximum (FWHM) is $18.1 \mathrm{meV}$ which is probably due to composition fluctuation in quaternary compound $(\mathrm{Cd}, \mathrm{Mn}, \mathrm{Mg}) \mathrm{Te}$ material. PL peak of QDs consist of many quite sharp emission lines, as expected for QDs. However, they are not very well resolved which makes them rather similar to wide QW peak at higher energy than to QD peak observed for similar $\mathrm{CdTe} /(\mathrm{Cd}, \mathrm{Mg}) \mathrm{Te}$ QDs grown in samples without QWs [20], where resolving individual QDs was not difficult. Most probably, coupling of QDs and QW is responsible by increased broadening of individual QD lines. 
The second system is proposed for fabrication of resonant structures, and implies the same energy of QW and QDs. In this systems we use binary ZnTe QW, barrier made of $(\mathrm{Zn}, \mathrm{Mg}) \mathrm{Te}$ and QDs made of CdTe (see Fig. 2a). Both PL and reflectivity spectra (Fig. 2b,c) show that $(\mathrm{Zn}, \mathrm{Mg}) \mathrm{Te}$ barrier is not very high - only $0.1 \mathrm{eV}$ above energy of QW. This corresponds to about $10 \%$ of $\mathrm{Mg}$ in the barrier. Comparing to previous system, PL peak of QDs is much broader and resolving of individual QD lines is easier. On the other hand, binary $\mathrm{ZnTe} \mathrm{QW}$ has re- latively narrow PL peak with FWHM equal to $7.9 \mathrm{meV}$. Both properties: wide QD peak and narrow QW peak should be consider as an advantage for future optical spectroscopy investigations. Moreover, it is possible to find QDs exactly in resonance with a QW peak. One of the samples contains no QW which provides additional piece of information - the peak related to $\mathrm{QW}$ is visible only in one sample, while luminescence of QDs is visible in both samples.
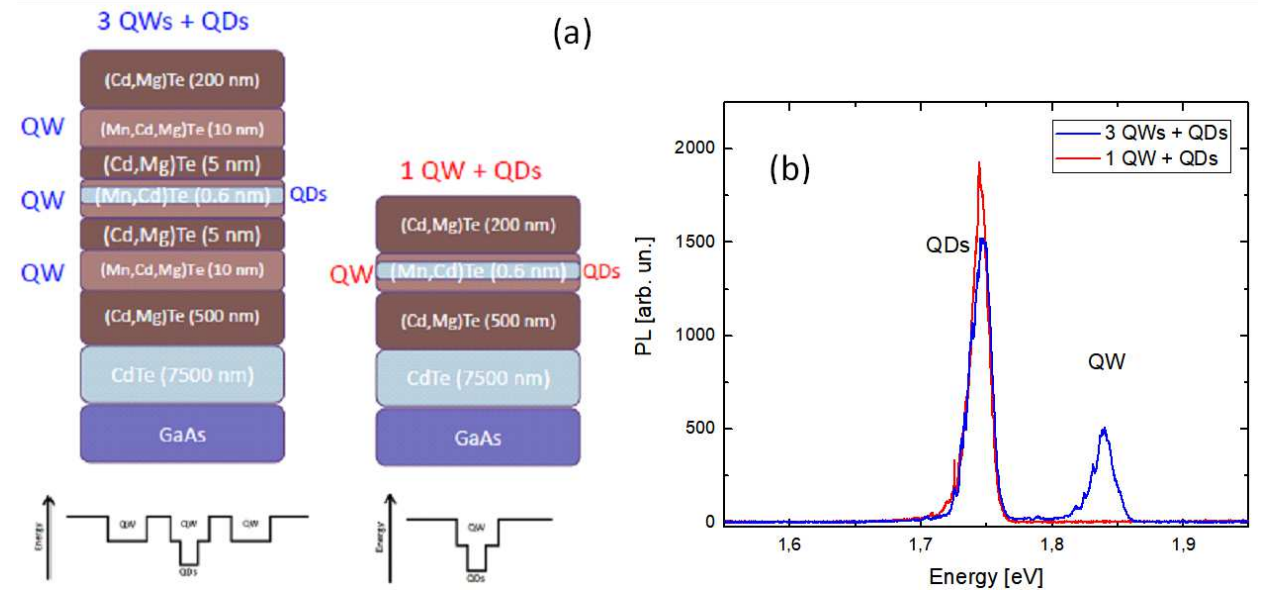

Fig. 1. (a) Scheme of structures with (Cd,Mn, Mg)Te QWs containing $\mathrm{Mg}$ both in QW and in barrier material. In one of QWs there are CdTe QDs. Below, scheme of conduction band is shown. (b) PL spectra showing emission from QDs and QWs, but if there is only a QW containing QDs inside, it is not emitting, because carriers are effectively transferred to QDs. Measurement at $T=8 \mathrm{~K}$. PL is excited by $405 \mathrm{~nm}$ laser.
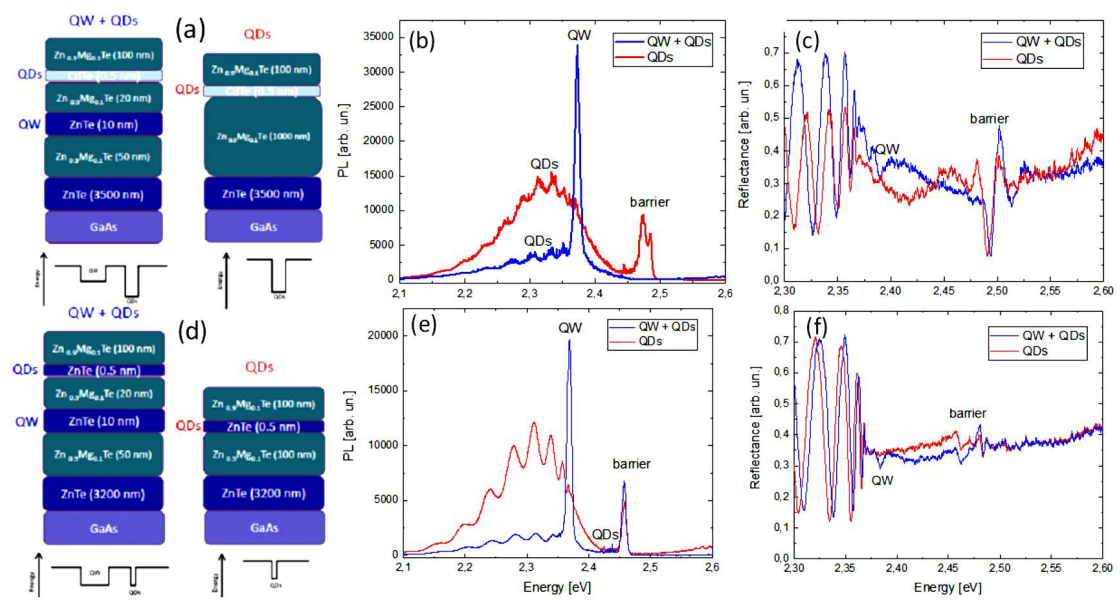

Fig. 2. Two systems based on (Zn,Mg)Te barriers. (a) and (d) - scheme of structures and below scheme of conduction band. In (a) structures with CdTe QDs and ZnTe QWs, in (d) structures with ZnTe QDs and ZnTe QWs. (b,e) PL and (c,f) reflectivity spectra of samples containing only QDs is compared with PL and reflectivity spectra of samples containing QW and QDs. This indicates identification of observed peaks. Measurement at $T=8 \mathrm{~K}$. PL is excited by $405 \mathrm{~nm}$ laser.

In the third system, we tested QDs and QW made of the same binary material (ZnTe) in the same barrier, $(\mathrm{Zn}, \mathrm{Mg}) \mathrm{Te}$ (Fig. 2d). As expected, in such a case most of carriers recombine in QW which is at lower energy due to smaller confinement (Fig. 2e). FWHM of PL peak for ZnTe QW is $8.6 \mathrm{meV}$. Sharp lines of QDs are observed very close to the barrier. It is possible that the peak at the energy close to energy of barrier band-gap is related to QDs, because the similar thin barrier (Fig. 2b) does not exhibit any PL peak. There is also visible a broad feature close to $2.3 \mathrm{eV}$, but it is not composed of sharp individual lines, and energy of this peak is too small for 
ZnTe QDs. Therefore we expect that such peak at $2.3 \mathrm{eV}$ is related to some kind of defects - probably located in the buffer, as similar broad PL is observed below QD lines of system 2. Intensity of this broad photoluminescence peak is modulated by interferences, which are well visible also in low energy part of reflectivity spectra.

\section{Conclusions}

Using molecular beam epitaxy we fabricated and investigated using low temperature optical spectroscopy three kinds of systems of coupled quantum wells and quantum dots: (i) (Cd,Mg,Mn)Te QWs at higher energy than CdTe:Mn QDs, (ii) resonant structures with ZnTe QWs and CdTe QDs, and (iii) ZnTe QDs at higher energy than ZnTe QWs. By analysis of photoluminescence and reflectivity spectra, we conclude that there is a possibility of effective carrier tunneling between structures, which opens possibility of subsequent testing of spin transfer efficiency.

\section{Acknowledgments}

The work was supported by the National Science Centre, Poland, under Project No. 2015/18/E/ST3/00559. Research was carried out with the use of CePT, CeZaMat and NLTK infrastructures financed by the European Union - the European Regional Development Fund within the Operational Programme "Innovative economy" for 2007-2013.

\section{References}

[1] F.K. Malinowski, F. Martins, P.D. Nissen, E. Barnes, Ł. Cywiński, M.S. Rudner, S. Fallahi, G.C. Gardner, M.J. Manfra, Ch.M. Marcus, F. Kuemmeth, Nat. Nanotechnol. 12, 16 (2017).

[2] M. Ściesiek, W. Pacuski, J.-G. Rousset, M. ParlińskaWojtan, A. Golnik, J. Suffczyński, Cryst. Growth Des. 17, 3716 (2017).

[3] T. Jakubczyk, H. Franke, T. Smoleński, M. Ściesiek, W. Pacuski, A. Golnik, R. Schmidt-Grund, M. Grundman, C. Kruse, D. Hommel, P. Kossacki, ACS Nano 8, 9970 (2014).

[4] Weng Qian-Chun, An Zheng-Hua, Xiong Da-Yuan, Zhu Zi-Qiang, Chin. Phys. Lett. 32, 108503 (2015).

[5] W.C. Fan, W.C. Chou, J.D. Lee, Ling Leb, Nguyen Dang Phu, Luc Huy Hoang, Physica B Condens. Matter, (2017).
[6] P. Kossacki, H. Boukari, M. Bertolini, D. Ferrand, J. Cibert, S. Tatarenko, J.A. Gaj, B. Deveaud, V. Ciulin, M. Potemski, Phys. Rev. B 70, 195337 (2004).

[7] P. Kossacki, D. Ferrand, M. Goryca, M. Nawrocki, W. Pacuski, W. Maślana, S. Tatarenko, J. Cibert, Physica E 32, 454 (2006).

[8] M. Goryca, D. Ferrand, P. Kossacki, M. Nawrocki, W. Pacuski, W. Maślana, J.A. Gaj, S. Tatarenko, J. Cibert, T. Wojtowicz, G. Karczewski, Phys. Rev. Lett. 102, 046408 (2009).

[9] J. Suffczyński, K. Kowalik, T. Kazimierczuk, A. Trajnerowicz, M. Goryca, P. Kossacki, A. Golnik, M. Nawrocki, J.A. Gaj, G. Karczewski, Phys. Rev. B 77, 245306 (2008).

[10] M. Goryca, T. Kazimierczuk, M. Nawrocki, A. Golnik, J.A. Gaj, P. Kossacki, P. Wojnar, G. Karczewski, Phys. Rev. Lett. 103, 087401 (2009).

[11] J. Kobak, T. Smoleński, M. Goryca, M. Papaj, K. Gietka, A. Bogucki, M. Koperski, J.-G. Rousset, J. Suffczyński, E. Janik, M. Nawrocki, A. Golnik, P. Kossacki, W. Pacuski, Nat. Commun. 5, 3191 (2014).

[12] C. Le Gall, L. Besombes, H. Boukari, R. Kolodka, J. Cibert, H. Mariette, Phys. Rev. Lett. 102, 127402 (2009).

[13] E. Baudin, E. Benjamin, A. Lemaître, O. Krebs, Phys. Rev. Lett. 107, 197402 (2011).

[14] T. Smoleński, W. Pacuski, M. Goryca, M. Nawrocki, A. Golnik, P. Kossacki, Phys. Rev. B 91, 045306 (2015).

[15] T. Smoleński, T. Kazimierczuk, J. Kobak, M. Goryca, A. Golnik, P. Kossacki, W. Pacuski, Nat. Commun. 7, 10484 (2016).

[16] A. Lafuente-Sampietro, H. Boukari, L. Besombes, Phys. Rev. B 95, 245308 (2017).

[17] A. Lafuente-Sampietro, H. Utsumi, H. Boukari, S. Kuroda, L. Besombes, Phys. Rev. B 95, 035303 (2017).

[18] J. Warnock, A. Petrou, R.N. Bicknell, N.C. GilesTaylor, D.K. Blanks, J.F. Schetzina, Phys. Rev. B 32, 8116 (1985).

[19] G. Karczewski, S. Maćkowski, M. Kutrowski, T. Wojtowicz, J. Kossut, Appl. Phys. Lett. 74, 3011 (1999).

[20] W. Pacuski, J.-G. Rousset, V. Delmonte, T. Jakubczyk, K. Sobczak, J. Borysiuk, K. Sawicki, E. Janik, J. Kasprzak, Cryst. Growth Des. 17, 2987 (2017). 